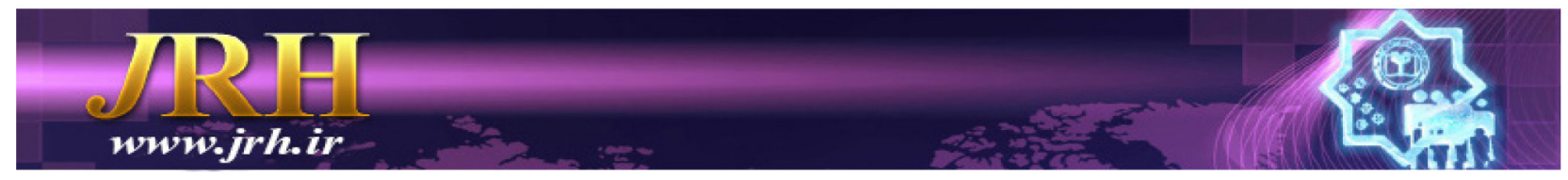

\title{
The compare psychological profiles of people based on MMPI-2's scales
}

Journal of Research \& Health Social Development \& Health Promotion Research Center

Vol. 7, No. 1, Jan \& Feb 2017 Pages: 551- 562

DOI: 10.18869/acadpub.jrh.7.1.551 Original Article

1. Correspondence to: Department of Family Health, Faculty of Family Institute, University of Shahid Beheshti, Tehran, Iran

E-mail: Mo_habibi@sbu.ac.ir

2. Department of Families with Special Needs, Faculty of Family Institute, University of Shahid Beheshti, Tehran, Iran 3. Department of Clinical Psychology, Faculty of Psychology and Educational Science, University of Kharazmi, Tehran, Iran

4. Department of Clinical Psychology, Faculty of Psychology and Educational Science, University of Tehran, Tehran, Iran

Received: 27 Jan 2014

Accepted: 12 Jul 2014

How to cite this article: Habibi M, Dehghani M, Jebreili H, Bahramy Monajemi M. The compare psychological profiles of people based on MMPI-2's scales. J Research \& Health2017; 7(1): 551- 562 .

\author{
Mojtaba Habibi ${ }^{1}$, Mohsen Dehghani ${ }^{2}$, Hashem Jebraeili ${ }^{3}$, Mani Bahrami
} Monajemi $^{4}$

\begin{abstract}
Considering the importance of screening and identifying people at risk of mental disorders in order to determine the prevalence of mental disorders and carry out activities to the prevention and treatment, the present study aimed to compare psychological profiles of people based on MMPI-2's scales according to birth order, age and educational level in general population of 18-80 years old. Current study is analytical- cross sectional. Statistical population included all Iranian people between 18 to 80 years old who had minimum 8 classes of education and had no history of mental illness or brain injury. Among this population, 1418 individuals were selected by ratio sampling method from Tehran, Isfahan, Mashhad, Tabriz and Shiraz cities and they were assessed by Minnesota Multiphase Personality Inventory (MMPI-2). Results showed there is significant difference between people according to their age in scales of L (Lying), D (Depression), Ma (Mania), Pt (Psychastenia), Sc(Schizophrenia), and Si(Social Introversion), and there was significant difference based on level of education in scales of $\mathrm{F}$ (Infrequency), K (Defensiveness), Pa (Paranoia), Pt (Psychastenia), Sc (Schizophrenia) and Si (Social Introversion). However, there was no significant differences between individuals based on birth order in validity and clinical scale of MMPI-2. Education and age are important factors which can influence mental health. It can be inferred that people with high level of education in comparison of people with low educational level have fewer mental disorders and higher mental wellbeing. Furthermore, people of different ages show different patterns of mental disorders.
\end{abstract}

Keywords: Age, Birth Order, Education, Psychological

\section{Introduction}

Based on 4th edition of DSM [1], mental disorder is syndrome or behavioral/psychological pattern which has clinical importance and afflicted individuals suffer from discomfort, disability and increased risk of pain, death, pain, disability and loss of freedom. This pattern or syndrome shouldn't be predictable or response in accordance with the culture of a particular event (for example, death of loved person). Additionally, this syndrome should be sign of psychological/biological or behavioral problems. It is more than 100 years that mental assessment is one the most dominant area in psychology which psychologists have employed a lot of 
tools in order to assess various psychological aspects such as intelligence, mood or clinical features. Psychological tools differ due to their implementation procedure, theoretical basics, their goals and efficacy [2]. In order to assess mental problems, various tools have been used so far such as morey personality assessment inventory [3] or millon clinical multiaxial inventory-III [4] that can be used specifically as specific goal and circumstances. One of the most prominent tools is Minnesota Multiphasic Personality Inventory (MMPI), which can be used in assessing wide area like mood states, anxiety, somatoform disorders or personality and thought disorders [5].

Evaluation psychiatric disorders in different populations indicates using different assessment tools of the demographic variables on the prevalence of the disorder

Birth order is one the variables which its role has been discussed with respect to psychological characteristics. For the first time in 1930, Alfred Adler [6] introduced this variable. Adler's work led to the birth and the researchers' attention on several variables such as intelligence $[7,8]$, progression [9], mental ability [10] and sexual orientation [11]. Discussions regarding birth order and its influence penetrated into the area of mental disorders and its influence on OCD [12,13], Schizophrenia [14], Sexual identity disorder [15] and delinquency [16] have been assessed thoroughly. Researches shows that due to higher expectation to first child, they have high rate of anxiety and guilty and have higher tendency toward psychological problems [17]. This claim has been supported vastly by different studies [18]. Although, in study of Denis and Colleagues [19] relationship of birth order and characteristics such as agreeableness, psychological stability, reliability, sociability and self-esteem were assessed. Analysis of the results showed that birth order has no effect on these variables in men or in women and hypotheses in this area further because of ethnic cultures and opinions in this case is lower [20].

Age is another demographic variables that could affect the prevalence psychiatric disorders. According to DSM, prevalence rate of psychological disorders may differ due to individuals' age. For instance, onset of Schizophrenia is mostly between late adolescence and middle of third decade of life and mean age for manic episode onset is mostly around age of 18 while older people are mostly struggling with neurocognitive disorders. Nonetheless, Springer and Colleagues [21] investigated this hypothesis that whether well-being and mental health changes over time or not? They focused in their study on psychological wellbeing in different ages through Ryff's model of psychological wellbeing (RPWB). The results of the study entailed the stability of psychological wellbeing of individuals in various sub-scales (personal development, positive relationships, purpose of life, self acceptance) through coarse of life except environmental mastery.

Level of education is one the influential factors which may affect psychological wellbeing $[22,23]$. Most of the studies have been suggested that higher education levels are associated with lower levels of depressive symptoms [24,25]. Furthermore, some other studies suggested that education can be influential in both triggering and fading of depression [26-28]. More importantly, based on cumulative advantage theory, mental resources correlate with level of education in a coarse of life. It means that individuals with higher level of education tend to use their resources in order to postpone psychological problems or even solve them [29].

In longitudinal study study, Koivusilta and colleagues [30] studied relationship between health and behaviors associated with the level of education. The result of their study showed that there is a significant positive relationship between health-related behaviors in adulthood between the ages of 6-12 years of age, and education level. In this study the relationship between health and education would facilitated through academic achievement and background variables such as educational success and appropriate demographic history. In another study conducted by Karmakar \& Breslin [31], they studied the influence of educational level and job features on mental 
hygiene among young individuals. The result of the study showed that job aspects of young people are directly related to their educational level and these two factors substantially influence the mental health of people.

As aforementioned, demographical variables assumed to be influential on mental wellbeing of individuals. Hence, occupying proper tool in order to assess mental states of individuals in order to implement proper therapeutic process is vital. Furthermore, lack of study in this area motivated us to conduct current study and provide an answer to this question: Is psychological profile of examinee based on birth-order, age and educational level in MMPI-2 is different or not?

\section{Method}

The study is cross-sectional. Current study can be categorized as applied study, because professionals and clinicians in therapeutic process can use its results. Findings of applied studies are mostly based on time and place such as this studywhich is suitable to cultural context. Statistical population of this study included all the Iranian youth between 18-80 years old who had at least 8 class of educational level and had no history of mental illness and brain injury. Sampling method was stratified sampling. Regarding sampling based on geographical distribution of Iran we divided Iran into 4 areas: 1) North, northwest and northeast, which included 234 persons. 2) Central west and west included 176 persons. 3) Center and south east of Iran included 350 persons. 4) Tehran and north regions included 512 participants. Among 1418 participants; 510 of them were male (36\%), $895(63.1 \%)$ were female and $13(0.9 \%)$ had no specified sexual orientation. Age range of this sample was between 18-80 years old. Among this group 504(35.5\%) were men, 875(61.7\%) were single and $39(2.8 \%)$ didn't have specific marital status. Regarding educational level, $83(5.9 \%)$ had secondary school educational level, 553(39\%) had high-school diploma, 64(4.5\%) had high-school upper-diploma, 584 (41.2\%) had bachelor degree, 134(9.4\%) had master degree and higher degrees. Among 487 individuals of married couple, 154(10.9\%) were without child, 104(7.3\%) had one child, $125(8.8 \%)$ had two children, $69(4.9 \%)$ had 3 children and $26(1.8 \%)$ had 4 or more children. Regarding age, 373 participants were in range of 16-19 years old, 461 people between 20-24 years old, 238 people between 25-29, 158 of people between $30-39,116$ of people between 40-49 and 70 people were more than fifty years old.

MMPI is standard questionnaire for summoning wide range of self-report specifications which scoring them will generate a quantitative index from emotional adaptation of participants and their prospects regarding attending the test. The mainframe of this test included 504 positive sentences which could be responded as correct and wrong. Later, by adding repetitive elements and 5th (Masculine/Feminine) scale and 0 (Social intervention) scale, number of questions were add to 566 items. In standard form of 1989, former mainframe of MMPI some of the elements were altered, eliminated and added by keeping . Consequently, number of item reached to 567. Original MMPI had 13 subscales which three subscales were about validity and 10 other scales were about clinical/personality indexes. In newer version of MMPI-2 and MMPI-A, 10 major clinical/ personality subscales and three original validity subscales were preserved but number of itemss was increased [32]. In this study only 370 first items of questionnaire were used which would allow us score the validity and clinical scales. In the present study data were analyzed by using SPSS-16 and by using multivariate analysis of variance (MANOVA).

\section{Results}

In order to assess the influence of birth order on MMPI-2's scales MANOVA test was used. Assumption of homogeneity of variancecovariance matrix approved by M-BOX's test $(\mathrm{F}=1.10, \mathrm{p}>0.05)$. Results of assessment the influence of birth-order variable by Lambday Wilks on linear combination of MMPI-2 sub-scales was demonstrative of simple nonmeaningful effect of birth-order variable $(\mathrm{F}=0.95, \mathrm{p}>0.05)$ on MMPI-2 scales. 
Table 1 Descriptive statistics of MMPI sub-scales in based on birth-order

\begin{tabular}{|c|c|c|c|c|c|c|c|c|c|}
\hline Scales & $\begin{array}{l}\text { Birth } \\
\text { order }\end{array}$ & M & SD & $\mathrm{N}$ & Scales & $\begin{array}{l}\text { Birth } \\
\text { order }\end{array}$ & M & SD & $\mathrm{N}$ \\
\hline \multirow{7}{*}{ Lying (L) } & First & 5.15 & 2.32 & 375 & \multirow{7}{*}{$\begin{array}{l}\text { Masculine/ } \\
\text { Feminine } \\
(\mathrm{M} / \mathrm{F})\end{array}$} & First & 28.36 & 5.01 & 375 \\
\hline & Second & 5.19 & 2.29 & 333 & & Second & 28.57 & 4.84 & 333 \\
\hline & Third & 5.38 & 2.37 & 269 & & Third & 28.43 & 4.86 & 269 \\
\hline & Forth & 5.25 & 2.05 & 165 & & Forth & 28.19 & 4.55 & 165 \\
\hline & Fifth & 5.01 & 2.11 & 103 & & Fifth & 28.22 & 4.77 & 103 \\
\hline & $\begin{array}{c}\text { Sixth } \\
\text { and more }\end{array}$ & 5.39 & 2.27 & 159 & & $\begin{array}{c}\text { Sixth } \\
\text { and more }\end{array}$ & 27.84 & 4.35 & 159 \\
\hline & Total & 5.23 & 2.27 & 1404 & & Total & 28.33 & 4.79 & 1404 \\
\hline \multirow{7}{*}{ Infrequency $(\mathrm{F})$} & First & 12.08 & 7.36 & 375 & \multirow{7}{*}{ Paranoia $(\mathrm{Pa})$} & First & 14.51 & 4.45 & 375 \\
\hline & Second & 11.68 & 7.15 & 333 & & Second & 14.22 & 4.65 & 333 \\
\hline & Third & 12.25 & 8.07 & 269 & & Third & 14.71 & 4.27 & 269 \\
\hline & Forth & 12.94 & 8.40 & 165 & & Forth & 14.73 & 4.60 & 165 \\
\hline & Fifth & 12.33 & 7.77 & 103 & & Fifth & 15.04 & 4.20 & 103 \\
\hline & $\begin{array}{c}\text { Sixth } \\
\text { and more }\end{array}$ & 13.04 & 7.66 & 159 & & $\begin{array}{c}\text { Sixth } \\
\text { and more }\end{array}$ & 14.05 & 3.89 & 159 \\
\hline & Total & 12.25 & 7.65 & 1404 & & Total & 14.49 & 4.41 & 1404 \\
\hline \multirow{7}{*}{$\begin{array}{l}\text { Defensiveness } \\
(\mathrm{K})\end{array}$} & First & 12.78 & 4.25 & 375 & \multirow{7}{*}{$\begin{array}{l}\text { Psychasthenia } \\
\text { (Ps) }\end{array}$} & First & 20.64 & 8.58 & 375 \\
\hline & Second & 13.30 & 4.63 & 333 & & Second & 19.70 & 9.40 & 333 \\
\hline & Third & 12.93 & 4.51 & 269 & & Third & 20.28 & 8.56 & 269 \\
\hline & Forth & 13.37 & 4.22 & 165 & & Forth & 20.14 & 8.12 & 165 \\
\hline & Fifth & 12.65 & 3.99 & 103 & & Fifth & 20.78 & 8.66 & 103 \\
\hline & $\begin{array}{c}\text { Sixth } \\
\text { and more }\end{array}$ & 13.23 & 4.59 & 159 & & $\begin{array}{c}\text { Sixth } \\
\text { and more }\end{array}$ & 20.55 & 9.05 & 159 \\
\hline & Total & 13.04 & 4.41 & 1404 & & Total & 20.29 & 8.78 & 1404 \\
\hline \multirow{7}{*}{$\begin{array}{l}\text { Hypochondriasis } \\
\text { (Hs) }\end{array}$} & First & 10.55 & 4.89 & 375 & \multirow{7}{*}{$\begin{array}{l}\text { Schizophrenia } \\
\text { (Sc) }\end{array}$} & First & 25.59 & 11.06 & 375 \\
\hline & Second & 10.76 & 4.91 & 333 & & Second & 24.90 & 12.02 & 333 \\
\hline & Third & 10.64 & 4.77 & 269 & & Third & 25.57 & 11.14 & 269 \\
\hline & Forth & 10.79 & 4.70 & 165 & & Forth & 26.09 & 10.02 & 165 \\
\hline & Fifth & 10.33 & 5.06 & 103 & & Fifth & 26.57 & 10.76 & 103 \\
\hline & $\begin{array}{c}\text { Sixth } \\
\text { and more }\end{array}$ & 10.53 & 4.86 & 159 & & $\begin{array}{c}\text { Sixth } \\
\text { and more }\end{array}$ & 25.83 & 10.91 & 159 \\
\hline & Total & 10.63 & 4.85 & 1404 & & Total & 25.58 & 11.15 & 1404 \\
\hline \multirow{7}{*}{ Depression(D) } & First & 24.54 & 5.95 & 375 & \multirow{7}{*}{ Mania (Ma) } & First & 20.94 & 5.28 & 375 \\
\hline & Second & 24.08 & 5.86 & 333 & & Second & 20.61 & 5.39 & 333 \\
\hline & Third & 24.66 & 5.78 & 269 & & Third & 20.33 & 4.78 & 269 \\
\hline & Forth & 24.26 & 5.53 & 165 & & Forth & 20.64 & 4.74 & 165 \\
\hline & Fifth & 24.08 & 6.51 & 103 & & Fifth & 20.85 & 5.30 & 103 \\
\hline & $\begin{array}{c}\text { Sixth } \\
\text { and more }\end{array}$ & 24.16 & 5.81 & 159 & & $\begin{array}{c}\text { Sixth } \\
\text { and more }\end{array}$ & 21.30 & 5.27 & 159 \\
\hline & Total & 24.34 & 5.87 & 1404 & & Total & 20.75 & 5.15 & 1404 \\
\hline
\end{tabular}


Compare psychological profiles MMPI-2's scales

\begin{tabular}{|c|c|c|c|c|c|c|c|c|c|}
\hline & First & 23.63 & 5.87 & 375 & & First & 30.01 & 7.44 & 375 \\
\hline & Second & 24.13 & 5.25 & 333 & & Second & 28.95 & 7.52 & 333 \\
\hline & Third & 23.68 & 5.51 & 269 & & Third & 30.52 & 7.40 & 269 \\
\hline \multirow[t]{7}{*}{ Hysteria (Hy) } & Forth & 24.08 & 5.33 & 165 & $\begin{array}{c}\text { Social } \\
\text { Introversion } \\
\text { (Si) }\end{array}$ & Forth & 29.93 & 6.563 & 165 \\
\hline & Fifth & 22.85 & 6.22 & 103 & & Fifth & 29.77 & 8.017 & 103 \\
\hline & $\begin{array}{c}\text { Sixth } \\
\text { and more }\end{array}$ & 23.31 & 5.62 & 159 & & $\begin{array}{c}\text { Sixth } \\
\text { and more }\end{array}$ & 29.76 & 6.60 & 159 \\
\hline & Total & 23.72 & 5.60 & 1404 & & Total & 29.80 & 7.31 & 1404 \\
\hline & First & 20.85 & 5.51 & 375 & & & & & \\
\hline & Second & 20.53 & 5.94 & 333 & & & & & \\
\hline & Third & 20.80 & 5.68 & 269 & & & & & \\
\hline \multirow[t]{4}{*}{$\begin{array}{l}\text { Psychopathic } \\
\text { Deviation (Pd) }\end{array}$} & Forth & 20.80 & 5.16 & 165 & & & & & \\
\hline & Fifth & 20.54 & 5.95 & 103 & & & & & \\
\hline & $\begin{array}{c}\text { Sixth } \\
\text { and more }\end{array}$ & 20.04 & 5.20 & 159 & & & & & \\
\hline & Total & 20.65 & 5.60 & 1404 & & & & & \\
\hline
\end{tabular}

In order to assess the influence of educational levels on MMPI-2's scales MANOVA test was used. Assumption of homogeneity of variancecovariance matrix approved by M-BOX's test $(\mathrm{F}=1.10, \mathrm{p}>0.05$ ). Results of assessment the influence of educational levels variable by Lambday Wilks on linear combination of
MMPI-2 sub-scales was demonstrative of simple meaningful effect of educational levels variable $(\mathrm{F}=2.72, \mathrm{p}<0.05)$ on MMPI-2 scales. Based on results on Table 3 , it is obvious that there is meaningful difference based on educational level in scales of lying, infrequency, defensiveness, psychopathy, Masculine/

Table 2 Descriptive statistics of MMPI sub-scales in based on educational levels

\begin{tabular}{|c|c|c|c|c|c|c|c|c|c|}
\hline Scales & $\begin{array}{l}\text { Educational } \\
\text { levels }\end{array}$ & M & SD & $\mathrm{N}$ & Scales & $\begin{array}{l}\text { Educational } \\
\text { levels }\end{array}$ & M & SD & $\mathrm{N}$ \\
\hline \multirow{6}{*}{ Lying (L) } & $\begin{array}{c}\text { Secondary } \\
\text { School } \\
\text { Degree }\end{array}$ & 4.84 & 2.12 & 83 & \multirow{6}{*}{$\begin{array}{l}\text { Masculine/ } \\
\text { Feminine } \\
(\mathrm{M} / \mathrm{F})\end{array}$} & $\begin{array}{c}\text { Secondary } \\
\text { School } \\
\text { Degree }\end{array}$ & 27.63 & 4.19 & 83 \\
\hline & Diploma & 5.35 & 2.34 & 552 & & Diploma & 27.70 & 4.84 & 552 \\
\hline & $\begin{array}{l}\text { Upper } \\
\text { Diploma }\end{array}$ & 5.94 & 2.74 & 64 & & $\begin{array}{c}\text { Upper } \\
\text { Diploma }\end{array}$ & 28.80 & 4.39 & 64 \\
\hline & Bachelor & 5.07 & 2.14 & 579 & & Bachelor & 29.01 & 4.71 & 579 \\
\hline & $\begin{array}{l}\text { Master } \\
\text { and Upper } \\
\text { Degrees }\end{array}$ & 5.37 & 2.28 & 126 & & $\begin{array}{l}\text { Master } \\
\text { and Upper } \\
\text { Degrees }\end{array}$ & 28.28 & 5.16 & 126 \\
\hline & Total & 5.23 & 2.27 & 1404 & & Total & 28.33 & 4.79 & 1404 \\
\hline
\end{tabular}




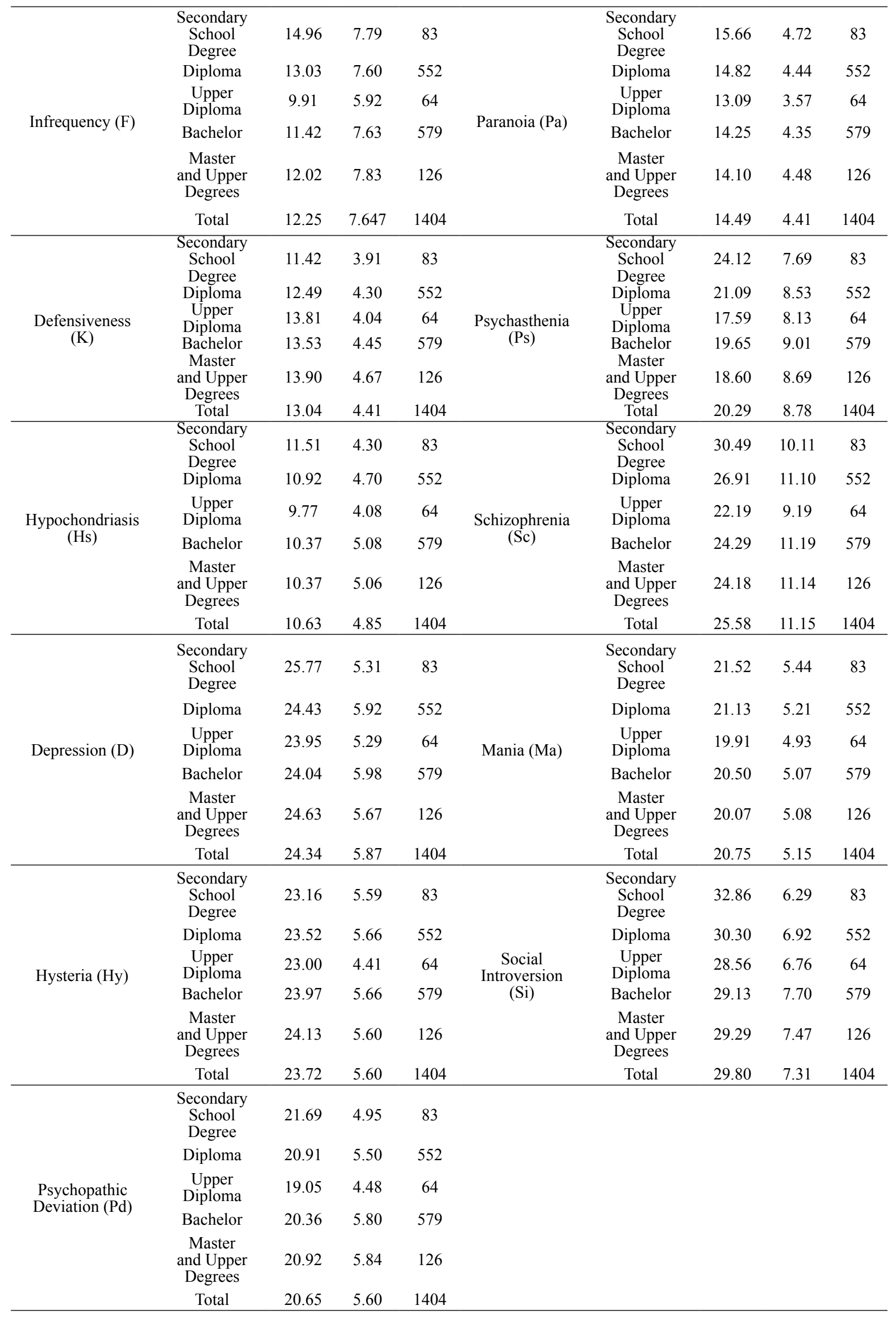


Compare psychological profiles MMPI-2's scales

Table 3 ANOVA test for assessment the influence of educational levels on MMPI-2 Scales

\begin{tabular}{|c|c|c|c|c|c|c|}
\hline $\begin{array}{l}\text { Source of } \\
\text { variation }\end{array}$ & Dependant Variable & Type III SS & $\mathrm{df}$ & MS & $\mathrm{F}$ & $\mathrm{p}$ \\
\hline \multirow{13}{*}{$\begin{array}{l}\text { Educational } \\
\quad \text { level }\end{array}$} & Lying & 69.57 & 4 & 17.39 & 3.40 & .009 \\
\hline & Infrequency & 1700.76 & 4 & 425.19 & 7.41 & .001 \\
\hline & Defensiveness & 652.28 & 4 & 163.07 & 8.57 & .001 \\
\hline & Hypochondriasis & 205.00 & 4 & 51.25 & 2.19 & .068 \\
\hline & Depression & 247.58 & 4 & 61.90 & 1.80 & .126 \\
\hline & Hysteria & 139.90 & 4 & 34.97 & 1.12 & .347 \\
\hline & $\begin{array}{l}\text { Psychopathic } \\
\text { deviation }\end{array}$ & 347.84 & 4 & 86.96 & 2.79 & .025 \\
\hline & Masculine/Feminine & 541.42 & 4 & 135.36 & 5.97 & .001 \\
\hline & Paranoid & 348.42 & 4 & 87.10 & 4.53 & .001 \\
\hline & Psychastenia & 2635.76 & 4 & 658.94 & 8.74 & .001 \\
\hline & Schizophrenia & 4920.41 & 4 & 1230.10 & 10.16 & .001 \\
\hline & Manic & 268.11 & 4 & 67.03 & 2.54 & .039 \\
\hline & Social Introversion & 1303.18 & 4 & 325.79 & 6.18 & .001 \\
\hline \multirow{13}{*}{ Error Effect } & Lying & 7155.80 & 1399 & 5.12 & & \\
\hline & Infrequency & 80332.96 & 1399 & 57.42 & & \\
\hline & Defensiveness & 26609.15 & 1399 & 19.02 & & \\
\hline & Hypochondriasis & 32809.94 & 1399 & 23.45 & & \\
\hline & Depression & 48090.32 & 1399 & 34.38 & & \\
\hline & Hysteria & 43829.98 & 1399 & 31.33 & & \\
\hline & $\begin{array}{c}\text { Psychopathic } \\
\text { deviation }\end{array}$ & 43615.23 & 1399 & 31.18 & & \\
\hline & Masculine/Feminine & 31702.91 & 1399 & 22.66 & & \\
\hline & Paranoid & 26906.48 & 1399 & 19.23 & & \\
\hline & Psychastenia & 105460.83 & 1399 & 75.38 & & \\
\hline & Schizophrenia & 169433.65 & 1399 & 121.11 & & \\
\hline & Mania & 36984.60 & 1399 & 26.44 & & \\
\hline & Social Introversion & 73749.78 & 1399 & 52.72 & & \\
\hline
\end{tabular}

Feminine, paranoia, psychastenia, schizophrenia, mania, and social introversion. In order to confirm the results, scheffe test was conducted. The results were demonstrative that between people with secondary school degree and upper degrees, there were meaningful differences in scales of infrequency, defensiveness, psychastenia, schizophrenia, and social introversion.

In order to assess the influence of age factor on MMPI-2's scales MANOVA test was used. Assumption of homogeneity of variancecovariance matrix approved by M-BOX's test $(\mathrm{F}=1.10, \mathrm{p}>0.056)$. Results of assessment the influence of age variable by Lambday Wilks on linear combination of MMPI-2 sub-scales was demonstrative of simple meaningful effect of age variable $(\mathrm{F}=2.78, \mathrm{p}<0.05)$ on MMPI- 2 scales.
Based on a results on Table 5, it is obvious that there are meaningful differences between people in various age ranges in Scales of Lying, Infrequency, Defensiveness, Depression, Psychastenia, Schizophrenia, and Mania. In order to confirm the results, Scheffe test was conducted. The results showed that between people between 16-19 years old and more than 40 years old there were meaningful differences in scales of lying, depression, manic, schizophrenia, psychastenia and social introversion.

\section{Discussion}

Current study was conducted in order to compare the psychological profile of participants by MMPI-2 subscales based 
Table 4 Descriptive statistics of MMPI sub-scales in based on age

\begin{tabular}{|c|c|c|c|c|c|c|c|c|c|}
\hline Scales & Age & M & SD & $\mathrm{N}$ & Scales & Age & $\mathrm{M}$ & SD & $\mathrm{N}$ \\
\hline \multirow{7}{*}{ Lying (L) } & 16-19 & 4.95 & 2.28 & 372 & \multirow{7}{*}{$\begin{array}{l}\text { Masculine/ } \\
\text { Feminine } \\
(\mathrm{M} / \mathrm{F})\end{array}$} & $16-19$ & 28.06 & 4.84 & 372 \\
\hline & $20-24$ & 5.14 & 2.24 & 458 & & $20-24$ & 28.69 & 4.85 & 458 \\
\hline & $25-29$ & 4.98 & 2.01 & 237 & & $25-29$ & 28.63 & 4.87 & 237 \\
\hline & $30-39$ & 5.68 & 2.31 & 158 & & $30-39$ & 27.91 & 4.54 & 158 \\
\hline & $40-49$ & 5.86 & 2.45 & 116 & & $40-49$ & 28.17 & 4.52 & 116 \\
\hline & $\begin{array}{l}\text { More } \\
\text { than } 50\end{array}$ & 6.22 & 2.34 & 63 & & $\begin{array}{l}\text { More } \\
\text { than } 50\end{array}$ & 27.59 & 4.87 & 63 \\
\hline & Total & 5.23 & 2.27 & 1404 & & Total & 28.33 & 4.79 & 1404 \\
\hline \multirow{7}{*}{ Infrequency $(\mathrm{F})$} & $16-19$ & 13.58 & 7.86 & 372 & \multirow{7}{*}{ Paranoia $(\mathrm{Pa})$} & $16-19$ & 14.67 & 4.34 & 372 \\
\hline & $20-24$ & 11.91 & 7.76 & 458 & & $20-24$ & 14.48 & 4.43 & 458 \\
\hline & $25-29$ & 11.99 & 7.22 & 237 & & $25-29$ & 14.38 & 4.29 & 237 \\
\hline & $30-39$ & 11.85 & 7.74 & 158 & & $30-39$ & 14.39 & 4.74 & 158 \\
\hline & $40-49$ & 11.18 & 7.43 & 116 & & $40-49$ & 14.35 & 4.51 & 116 \\
\hline & $\begin{array}{c}\text { More } \\
\text { than } 50\end{array}$ & 10.68 & 6.38 & 63 & & $\begin{array}{l}\text { More } \\
\text { than } 50\end{array}$ & 14.46 & 4.17 & 63 \\
\hline & Total & 12.25 & 7.68 & 1404 & & Total & 14.49 & 4.41 & 1404 \\
\hline \multirow{7}{*}{$\begin{array}{l}\text { Defensiveness } \\
(\mathrm{K})\end{array}$} & 16-19 & 12.70 & 4.31 & 372 & \multirow{7}{*}{$\begin{array}{l}\text { Psychasthenia } \\
\text { (Ps) }\end{array}$} & 16-19 & 21.15 & 8.50 & 372 \\
\hline & $20-24$ & 13.07 & 4.44 & 458 & & $20-24$ & 20.42 & 8.89 & 458 \\
\hline & $25-29$ & 12.68 & 4.36 & 237 & & $25-29$ & 20.26 & 8.88 & 237 \\
\hline & $30-39$ & 13.27 & 4.38 & 158 & & $30-39$ & 19.70 & 8.83 & 158 \\
\hline & $40-49$ & 13.54 & 4.69 & 116 & & $40-49$ & 19.18 & 9.34 & 116 \\
\hline & $\begin{array}{l}\text { More } \\
\text { than } 50\end{array}$ & 14.75 & 4.14 & 63 & & $\begin{array}{l}\text { More } \\
\text { than } 50\end{array}$ & 17.84 & 7.89 & 63 \\
\hline & Total & 13.04 & 4.41 & 1404 & & Total & 20.29 & 8.78 & 1404 \\
\hline \multirow{7}{*}{$\begin{array}{l}\text { Hypochondriasis } \\
\text { (Hs) }\end{array}$} & $16-19$ & 10.44 & 4.55 & 372 & \multirow{7}{*}{$\begin{array}{l}\text { Schizophrenia } \\
\text { (Sc) }\end{array}$} & $16-19$ & 27.22 & 10.94 & 372 \\
\hline & $20-24$ & 10.40 & 5.00 & 458 & & $20-24$ & 25.29 & 11.06 & 458 \\
\hline & $25-29$ & 10.78 & 4.76 & 237 & & $25-29$ & 25.57 & 11.19 & 237 \\
\hline & $30-39$ & 10.70 & 5.35 & 158 & & $30-39$ & 24.71 & 11.44 & 158 \\
\hline & $40-49$ & 11.14 & 4.52 & 116 & & $40-49$ & 23.99 & 11.71 & 116 \\
\hline & $\begin{array}{l}\text { More } \\
\text { than } 50\end{array}$ & 11.60 & 5.06 & 63 & & $\begin{array}{l}\text { More } \\
\text { than } 50\end{array}$ & 23.14 & 10.15 & 63 \\
\hline & Total & 10.63 & 4.85 & 1404 & & Total & 25.58 & 11.15 & 1404 \\
\hline \multirow{7}{*}{ Depression (D) } & $16-19$ & 23.45 & 5.67 & 372 & \multirow{7}{*}{ Mania(Ma) } & $16-19$ & 21.54 & 5.20 & 372 \\
\hline & $20-24$ & 23.92 & 5.77 & 458 & & $20-24$ & 20.84 & 5.21 & 458 \\
\hline & $25-29$ & 24.73 & 5.86 & 237 & & $25-29$ & 20.81 & 4.89 & 237 \\
\hline & $30-39$ & 24.99 & 5.96 & 158 & & $30-39$ & 20.19 & 5.19 & 158 \\
\hline & $40-49$ & 26.08 & 5.56 & 116 & & $40-49$ & 19.37 & 4.98 & 116 \\
\hline & $\begin{array}{l}\text { More } \\
\text { than } 50\end{array}$ & 26.35 & 6.85 & 63 & & $\begin{array}{l}\text { More } \\
\text { than } 50\end{array}$ & 19.03 & 4.76 & 63 \\
\hline & Total & 24.34 & 5.87 & 1404 & & Total & 20.75 & 5.15 & 1404 \\
\hline \multirow{7}{*}{ Hysteria (Hy) } & $16-19$ & 23.40 & 5.45 & 372 & \multirow{7}{*}{$\begin{array}{c}\text { Social } \\
\text { Introversion } \\
\text { (Si) }\end{array}$} & $16-19$ & 29.59 & 7.12 & 372 \\
\hline & $20-24$ & 23.60 & 5.58 & 458 & & $20-24$ & 29.49 & 7.42 & 458 \\
\hline & $25-29$ & 24.15 & 5.59 & 237 & & $25-29$ & 29.92 & 7.92 & 237 \\
\hline & $30-39$ & 23.42 & 5.69 & 158 & & $30-39$ & 30.70 & 6.91 & 158 \\
\hline & $40-49$ & 23.89 & 5.69 & 116 & & $40-49$ & 30.23 & 6.96 & 116 \\
\hline & $\begin{array}{l}\text { More } \\
\text { than } 50\end{array}$ & 25.32 & 6.01 & 63 & & $\begin{array}{l}\text { More } \\
\text { than } 50\end{array}$ & 29.86 & 6.93 & 63 \\
\hline & Total & 23.72 & 5.60 & 1404 & & Total & 29.80 & 7.31 & 1404 \\
\hline
\end{tabular}




\begin{tabular}{ccccc}
\hline & $16-19$ & 20.91 & 5.34 & 372 \\
& $20-24$ & 20.57 & 5.87 & 458 \\
& $25-29$ & 20.97 & 5.65 & 237 \\
Psychopathic & $30-39$ & 20.73 & 5.56 & 158 \\
Deviation (Pd) & $40-49$ & 19.55 & 5.17 & 116 \\
& More & 20.19 & 5.61 & 63 \\
& than 50 & 20.65 & 5.60 & 1404 \\
\hline
\end{tabular}

Table 5 ANOVA test for assess the influence of age range on MMPI-2 scales

\begin{tabular}{|c|c|c|c|c|c|c|}
\hline $\begin{array}{l}\text { Source of } \\
\text { variation }\end{array}$ & Dependant Variable & $\begin{array}{l}\text { Type III } \\
\text { SS }\end{array}$ & df & MS & $\mathrm{F}$ & $\mathrm{p}$ \\
\hline \multirow{13}{*}{ Age } & Lying & 188.00 & 5 & 37.60 & 7.47 & 0.001 \\
\hline & Infrequency & 1039.29 & 5 & 207.86 & 3.59 & 0.003 \\
\hline & Defensiveness & 295.35 & 5 & 59.07 & 3.06 & 0.009 \\
\hline & Hypochondriasis & 132.56 & 5 & 26.51 & 1.13 & 0.344 \\
\hline & Depression & 1080.17 & 5 & 216.04 & 6.39 & 0.001 \\
\hline & Hysteria & 267.22 & 5 & 53.44 & 1.71 & 0.129 \\
\hline & $\begin{array}{l}\text { Psychopathic } \\
\text { deviation }\end{array}$ & 207.52 & 5 & 41.51 & 1.33 & 0.250 \\
\hline & $\begin{array}{l}\text { Masculine/ } \\
\text { Feminine }\end{array}$ & 172.00 & 5 & 34.40 & 1.50 & 0.187 \\
\hline & Paranoid & 19.79 & 5 & 3.96 & .20 & 0.961 \\
\hline & Psychastenia & 858.79 & 5 & 171.76 & 2.24 & 0.048 \\
\hline & Schizophrenia & 1830.21 & 5 & 366.04 & 2.97 & 0.011 \\
\hline & Manic & 693.27 & 5 & 138.65 & 5.30 & 0.001 \\
\hline & Social Introversion & 212.92 & 5 & 42.58 & .80 & 0.553 \\
\hline \multirow{13}{*}{ Error Effect } & Lying & 7037.37 & 1398 & 5.03 & & \\
\hline & Infrequency & 80994.43 & 1398 & 57.94 & & \\
\hline & Defensiveness & 26966.09 & 1398 & 19.29 & & \\
\hline & Hypochondriasis & 32882.38 & 1398 & 23.52 & & \\
\hline & Depression & 47257.72 & 1398 & 33.80 & & \\
\hline & Hysteria & 43702.65 & 1398 & 31.26 & & \\
\hline & $\begin{array}{l}\text { Psychopathic } \\
\text { deviation }\end{array}$ & 43755.54 & 1398 & 31.30 & & \\
\hline & $\begin{array}{l}\text { Masculine/ } \\
\text { Feminine }\end{array}$ & 32072.33 & 1398 & 22.94 & & \\
\hline & Paranoid & 27235.11 & 1398 & 19.48 & & \\
\hline & Psychastenia & 107237.81 & 1398 & 76.71 & & \\
\hline & Schizophrenia & 172523.85 & 1398 & 123.41 & & \\
\hline & Mania & 36559.45 & 1398 & 26.15 & & \\
\hline & Social Introversion & 74840.04 & 1398 & 53.53 & & \\
\hline
\end{tabular}

on birth-order, educational level and age. As aforementioned by using Lambday Wilks on linear combination of MMPI-2 sub-scales is demonstrative of simple meaningful influences of age and educational level on subscales. Additionally, using MANOVA in order to assess simple influences of age and educational level on sub-scales of MMPI-2 illustrated that between people with varied educational level
(Secondary school, diploma, upper diploma, bachelor, master degree and upper degrees), there are meaningful differences in scales of Lying(L), Infrequency (K), Defensiveness (D), Hypochondriasis (Hs), Depression (D), Hysteria(Hy), Psychopathic deviation(Pd), Masculine/Feminine(M/F), Paranoid(Pa), Psychasthenia(Ps), Schizophrenia(Sc), Manic (Ma) and Social Introversion(Si) and regarding 
age range, there is a meaningful difference between age ranges $(16-19,20-50)$ in Scales of Lying (L), Infrequency (F), Defensiveness (K), Psychopathic deviation (Pd), Masculine/Feminine (M/F), Paranoid (Pa), Psychastenia(Pt), Schizophrenia $(\mathrm{Sc})$, Manic $(\mathrm{Ma})$, Social Introversion (Si). Although, difference between people regarding of birth order was not significant in our study but these results differ from the results of the study conducted by Ansari and colleagues [12], Conger and Conger [17], Zuckerman and Kuhlman [18]. The result of current study in case of birth order is similar to the result of study of Denis and colleagues [19].

Results of Scheffe test showed that the main difference is between people with secondary school level and people with upper degrees in scales of infrequencies, defensiveness, paranoid, psychastenia, schizophrenia and social introversion. People with secondary school degree, had higher score in scales of Infrequency(F), Paranoid(Pa), Psychastenia(Pt), Schizophrenia(Sc) and Social introversion(Si) and lower score in Defensiveness(K) comparing to people with higher degrees.

Results of clinical sub-scales indicated that people with lower educational level tend to experience more psychological discomfort and are more on verge of psychological disorders. These results are similar to the results of the studies conducted by Arja and Andres [30] Karamkar and Breslin [31] who announced high correlation between educational level and mental health and according to cumulative advantage theory [29] this can be caused by higher intellectual resources of people with higher education.

Results of validity scales are demonstrating negative correlation of educational level with infrequency and positive correlation of it with defensiveness. It means that people with higher educational level comparing to those with lower educational level, tend to have higher score in infrequency scale and lower score in defensiveness. Infrequency scale measures oddness and uncommon answers. Higher score in this scale is showing that person has not paid enough attention in answering the question. As the designers of this test claim, infrequency scale has a direct relation to level of education and it can be used in testing the literacy. Defensiveness scale is deligate scalewhich will demonstrate the efforts which participants tend to put in order to hide their psychological problems or manifesting good/bad projection of themselves or dramatic impression. High score in this scale showed defensiveness whereas lower score shows frankness and having self-criticism attitude toward oneself. Further researches [33] show that, this scale has relatoionship with educational level and social/financial status. It means that people with lower social status/educational level tend to have lower score in this scale.

Results of Scheffe test showed that regarding age's influence on sub scales of MMPI-2, majority of difference is between age group of 16-19 and a people older than 40 years old in scales of Lying (L), Depression (D), Manic (Ma), Psychastenia (Pt), Schizophrenia (Sc) and Social introversion (Si). Individuals in age rage of 16-19 comparing to individuals older than 40 in scales of Psychastenia (Pt), Schizophrenia (Sc) and Manic (Ma) had higher score and in scales of Lying (L), Depression (D) and Social introversion (Si) had lower score.

Findings of clinical subscales are similar to epidemiologic findings of mental disorders. Onset of Psychotic features of schizophrenia is between late adolescence and middle of $3 \mathrm{rd}$ decade of life and peak of first schizophrenia's onset in men is mostly in early/middle 3rd decade of life and in women is late 3 rd decade. Mean age of mania's onset is 18th year oflife[1]. Regarding existence of depression and social introversion in elderlies it is worth mentioning that although prevalence of depression in people between 18-29 is three times more than people older than 60 years old [1], but other superannuation disorders which have pseudo depression symptoms may caused higher score of elderlies in depression scale. Higher score of younger individuals in psychastenia scale can be caused by adverse financial circumstances of a country. Furthermore, stresses induced 
by adverse environment and unemployment can acte as an additional antagonistic factor and elevating psychastenia scale in younger individuals.

Findings rooted from validity scales demonstrated that younger individuals in comparison of older people had lower score in lying scale. It means that younger people tend to reveal themselves more that elderlies while elderlies try to demonstrate acceptable image of themselves.

\section{Conclusion}

Current findings releaved that demographic variables such as age, educational level are dominant factors in the prevalence and incidence rate of psychological disorders. As results of this study showed prevalence of psychological disorders is lower in people with higher educational level. Presumably, educated individuals have rich mental resources which can protect them from stresses and psychological discomforts. Trend of psychological disorders prevalence based on age varies. Acute psychological disorders are more prevalent in young ages and chronic psychological problems are more prevalence in older people. Thus, age is one of dominant factors, which should be included in diagnosis of mental disorders.

Generally, assessment methods of psychological disorder can be divided into three categories: 1.Interview 2.Test 3.Observation. Assessment tools should be valid and reliable. No single method will facilitate the process of psychopathology but each tool can be useful in assessing varied aspects of abnormality. When all of these methods amalgamate and precise, impeccable results have been generated, we can assume that scientific development has occurred. Hence, in future studies structured clinical interview based on DSM should be conducted. High number of questions, examinee tiredness and not cooperating during the test process worth mentioning as limitations of this study.

\section{Acknowledgments}

We are sincerely grateful of all the participants who helped us in conducting this research.

\section{Contributions}

Study design: MD, MH

Data collection and analysis: $\mathrm{MD}, \mathrm{MH}$

Manuscript preparation: MH, HJ, MBM

\section{Conflict of Interest}

"The author declare that they have no competing interests."

\section{Funding}

The author (s) received no financial support for the research, authorship and/or publication of this article.

\section{References}

1- American psychiatric association. Diagnostic and statistical manual of mental disorders, 4th Edn, Washington, D.C: APA; 1994.

2- Butcher J, Derksen J, Sloore H, Sirigatti S. Objective personality assessment of people in diverse cultures: european adaptations of the MMPI-2. Behav Res Ther2003; 41(7): 819-40.

3- Morey LC. Personality assessment inventory: professional manual. Odessa, FL: Psychological assessment resources, Inc; 1991.

4- Millon T, Millon C, Davis RD. Millon clinical multiaxial inventory-III. Minneapolis: National computer systems; 1994.

5- Hathaway SR, Mc Kinley JC. The minnesota multiphasic personality inventory. Minneapolis: University of minnesota press; 1943.

6-Adler A. The collected clinical works of Alfred Adler. Journal articles: 1931-1937. Birth order and early memories; social interest and education. Bellingham, WA: the classical adlerian translation project; 2005.

7- Burton D. Birth order and intelligence. J Soc Psychol1968; 76(2): 199-206.

8- Foster JW, Archer SJ. Birth order and intelligence; an immunological interpretation. Percept Mot Skills1976; 48: 79-93.

9- Kessler D. Birth order, family size and achievement. Family structure and wage determination. J Labor Econ1991; 9: 413-26.

10- Page EB, Grandon GM. Family configuration and mental ability: two theories contrasted with U.S. data. American Educational Research Journal1979; 16: 257-72. 
11- Bogart AF, Cairney J. The interaction of birth order and parental age on sexual orientation: an examination of two samples. J Biosoc Sci2004; 36(1): 19-37.

12- Ansari MA, Rehman RU, Siddiqui AS, Jabeen R. Correlation of obsessive compulsive disorder with birth order-one. JLUMHS2008; 129-132.

13- Khanna S, Channabasavanna SM. Birth order in obsessive compulsive disorder. Psychiatry Res1987; 21(4): 349-54.

14- Terzis A. Birth order, sex and Schizophrenia. Arq Neuropsiquiatr1986; 44(22): 147-54.

15- Zucker KJ, Lightbody S, Pecore K, Bradley SJ, Blanchard R. Birth order in girls with gender identity disorder. Eur Child Adolesc Psychiatry1998; 7(1): 30-5.

16- Cundiff PR. Ordered delinquency: The "effects" of birth order on delinquency. Pers Soc Psychol Bull2013; 39(8): 1017-29.

17- Conger RD, Conger KJ. Resilience in midwestern families: selected findings from the first decade of a prospective, longitudinal study. Journal of Marriage and Family2002; 64: 361-73.

18- Zuckerman M, Kuhlman DM. Personality and risktaking: common biosocial factors. J Pers2000; 68(6): 999-1029.

19- Denise D, Guastello DD, Guastello SJ. Birth category effects on the gordon personal profile variables. Journal for Articles in Support of the Null Hypothesis2002; 1(1): 1-7.

20- Hartmann P, Reuter M, Nyborg H. The relationship between date of birth and individual differences in personality and general intelligence: a large-scale study. Pers Individ Dif2006; 40(7): 1349-62.

21- Springer KW, Pudrovska T, Hauser RM. Does psychological well-being change with age? Longitudinal tests of age variations and further exploration of the multidimensionality of Ryff's model of psychological well-being. Soc Sci Res2011; 40(1): 392-8.

22- Mirowsky J, Ross CE. Education, social status, and health. New York: A.de gruyter; 2003.

23- Mirowsky J, Ross CE. Education and self-rated health: cumulative advantage and its rising importance. Res Agng2008; 30: 93-122.

24- Blazer DG, Moody Ayers S, Craft Morgan J, burchett

B. Depression in diabetes and obesity: racial/ethnic/ gender issues in older adults. $J$ psychosom Res2002; 53(4): 913-6.

25- Swenson CJ, Baxter J, Shetterly SM, Scarbro SL, Hamman RF. Depressive symptoms in hispanic and non-hispanic white rural elderly. The San Luis valley health and aging study. Am J Epidemiol2000; 152(11): 1048-55.
26- Grundy E, Glaser K. Socio-demographic differences in the onset and progression of disability in early old age: a longitudinal study. Age Ageing2000; 29(2): 14957.

27 Melzer H, Harrington R, Goodman R, Jenkins R. Children and adolescents who try to harm, hurt or kill themselves. London: Office for national statistics; 2001. 28- Zimmer Z, House JS. Education, income and functional limitation transitions among American adults: contrasting onset and progression. Int J Epidemiol2003; 32(6): 1089-97.

29- Ross CE, Chia-Ling W. The links between education and health. Am Sociol Rev1995; 60: 719-45.

30- Koivusilta L, Arja RA, Andres V. Health behaviors and health in adolescence as predictors of educational level in adulthood: a follow-up study from Finland. Soc Sci Med2003; 57: 577-93.

31- Karmakar SD, Breslin FC. The role of educational level and job characteristics on the health of young adults. Soc Sci Med2008; 66(9): 2011-22.

32- Grey Gras M. Psychological evaluation guidelines for clinical psychologists, psychiatrists and counselors. Translation publishing house Hasan Pasha Sharifi and Mohammad Reza Nykkho. Tehran: Growth; 2005.

33- Graham JR. MMPI-2: Assessing personality and psychopathology. New York: Oxford university press; 2000. 